\title{
What is the future of targeted therapy in rheumatology: biologics or small molecules?
}

\author{
Attila Mócsai ${ }^{1,2^{*}}$, László Kovács ${ }^{3+}$ and Péter Gergely ${ }^{4 \dagger}$
}

\begin{abstract}
Background: Until late in the 20th century, the therapy of rheumatic diseases relied on the use of drugs that had been developed through empirical approaches without detailed understanding of the molecular mechanisms involved. That approach changed with the introduction of biologic therapeutics at the end of the 20th century and by the recent development of small-molecule inhibitors of intracellular signal transduction pathways. Here we compare and discuss the advantages and disadvantages of those two groups of targeted anti-inflammatory therapeutics.

Discussion: TNF-blocking biologic agents were introduced into the therapy of rheumatoid arthritis and other autoimmune and inflammatory diseases in the late 1990s. Further biologic agents targeting cytokine networks or specific lymphocyte subsets have since been added to the armamentarium of anti-rheumatic therapy. During the last few years, another wave of novel discoveries led to the development of a new class of small molecule anti-inflammatory compounds targeting intracellular signal transduction molecules, such as tyrosine kinases. In all those cases, the specific targets of the drugs are well defined and significant knowledge about their role in the disease pathomechanism is available, qualifying them for being targeted therapeutics for inflammatory rheumatic diseases. While both groups of targeted therapeutics offer significant clinical benefit, they clearly differ in several aspects, such as the localization of their targets, their route of administration and target specificity, as well as technical details such as manufacturing procedures and cost basis. In this debate paper, we compare the advantages and disadvantages of the two different approaches, aiming to shed light on the possible future of targeted therapies.
\end{abstract}

Summary: Biologic therapeutics and small-molecule inhibitors both have significant advantages and disadvantages in the therapy of rheumatic diseases. The future of targeted therapies is one of the most exciting questions of current rheumatology research and therapy.

Keywords: Rheumatoid arthritis, Biologic therapies, TNF antagonists, Small molecule therapeutics, Kinase inhibitors, Tofacitinib

\section{Background}

Inflammatory rheumatic diseases such as rheumatoid arthritis (RA) or systemic lupus erythematosus (SLE) are severe and chronic diseases that affect a significant proportion of the population, cause deterioration of the quality of life and place a major burden on health care systems worldwide. The last several decades have

\footnotetext{
* Correspondence: mocsai.attila@med.semmelweis-univ.hu

${ }^{\dagger}$ Equal contributors

'Department of Physiology, Semmelweis University School of Medicine, 1094 Budapest, Hungary

2MTA-SE "Lendület" Inflammation Physiology Research Group of the Hungarian Academy of Sciences and the Semmelweis University, 1094 Budapest, Hungary

Full list of author information is available at the end of the article
}

witnessed significant improvement in the therapy of these diseases. However, there is an ongoing need for the development of newer, more efficient and more cost-effective therapeutic applications. The aim of this paper is to compare the benefits and drawbacks of two of the recent directions of targeted therapies in rheumatology: biologic therapeutics and small molecule inhibitors.

Steroids and non-steroidal anti-inflammatory drugs were introduced into the therapy of inflammatory rheumatic diseases around the middle of the 20th century (Figure 1) and provided symptomatic improvement and pain relief. However, they had significant side effects and, maybe more importantly, they failed to prevent disease progression and the development of a debilitating 


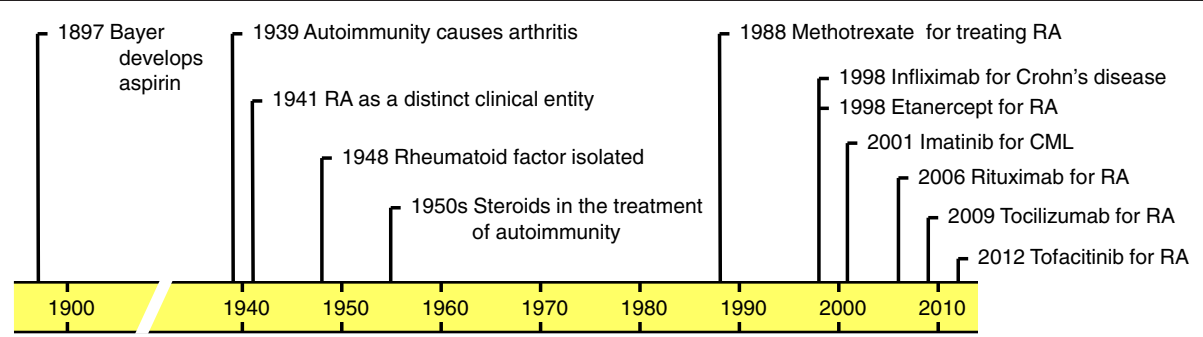

Figure 1 Timeline of rheumatoid arthritis research and therapy. RA, rheumatoid arthritis; CML, chronic myelogenous leukemia.

disease course. The identification of a beneficial effect of aminopterin antimetabolites led to the introduction of the chemotherapeutic agent methotrexate to the therapy of inflammatory rheumatic diseases in the late 1980s (Figure 1). The major advantage of methotrexate and related compounds (collectively termed disease-modifying anti-rheumatic drugs or DMARDs) was that they significantly delayed the progression of the diseases. Although methotrexate still remains the first line therapy for inflammatory rheumatic diseases, its cytotoxic (for example, hepatotoxic) nature and/or partial efficacy limit its use.

All of the above therapeutics were developed using an empirical approach without detailed understanding of their mechanism of action and therapeutic targets. However, the last decades have witnessed an explosion of our understanding of the inflammatory process and the molecular pathways involved. This led to a new approach of drug development whereby targeted therapies are developed by directly targeting molecules thought to be involved in the inflammatory process. A major approach for the development of targeted therapeutics has been the application of monoclonal antibody technologies for therapeutic purposes.

The first targeted therapeutics in immune mediated diseases were antibodies and related molecules interfering with the function of tumor necrosis factor- $\alpha$ (TNF- $\alpha$ or TNF), a major cytokine involved in various aspects of the autoimmune and inflammatory process [1]. The first TNFantagonists against inflammatory diseases were infliximab (first approved for the therapy of Crohn's disease) and etanercept (first approved for rheumatoid arthritis), both of them receiving marketing authorization in 1998 (Figure 1). Infliximab, as well as adalimumab, a third agent targeting the TNF- $\alpha$ cytokine, were later also approved for rheumatoid arthritis. Given the biologic, rather than chemical origin of these molecules, they have been termed biologic therapeutics. The major advance made by biologic agents was recognized by the 2003 Lasker award in clinical medicine given to Marc Feldmann and Ravinder Maini for 'the discovery of anti-TNF therapy as an effective treatment for rheumatoid arthritis and other autoimmune diseases' All of the above three drugs are proteinaceous molecules with antibody-related features. However, while infliximab and adalimumab are true monoclonal antibodies (infliximab is an engineered humanized mouse monoclonal antibody whereas adalimumab is a fully human antibody), etanercept is a fusion protein of the Fc region of a human immunoglobulin G (IgG) antibody linked to the extracellular portion of the human p75 TNF-receptor.

The initial advance with anti-TNF biologic therapeutics opened up new avenues for targeting other proinflammatory targets by biologic agents (Figure 1). Currently approved biologic agents for the treatment of rheumatoid arthritis include rituximab, a chimeric antibody targeting the CD20 receptor and, thus, leading to B-cell depletion; tocilizumab, a humanized mouse monoclonal antibody against IL-6; abatacept, a T-cell-blocking Fc-fusion protein of the extracellular domain of CTLA-4; and anakinra, an IL-1 receptor antagonist blocking the IL-1 receptor pathway [2]. There are also a large number of other biologic agents currently in advanced clinical trials for the therapy of rheumatoid arthritis $[2,3]$ and other immune mediated diseases.

The fact that all of the above biologic agents are of a proteinaceous nature (mainly antibodies or antibody-related molecules) determines several important features, such as route of administration, half-life, localization of the target, manufacturing standards, and so on (Table 1). Therefore, despite their rather different therapeutic targets, these biologic agents are considered a unique group of therapeutics in rheumatology.

During the last few years, an entirely different direction for developing novel anti-inflammatory agents has emerged. This was mainly driven by the tremendous success of targeting intracellular tyrosine kinases in cancer therapy. One of the prime examples of a breakthrough in oncology was the development of imatinib, an inhibitor of the Abl tyrosine kinase, for the targeted therapy of chronic myelogenous leukemia (Figure 1), a disease caused by the emergence of the BCR-Abl fusion protein through a unique chromosomal translocation [4]. Additional kinase inhibitors have also proved to provide significant clinical benefit in solid tumors [5]. Those developments also generated a vast experience with therapeutic targeting of intracellular kinases. However, kinases are not only involved in malignant processes but they also play critical roles in non-malignant (for example, inflammatory) diseases 
Table 1 Key characteristics of biologics versus small molecules

\begin{tabular}{lll}
\hline & Biologics & Small molecules \\
\hline Chemical composition & Protein & Organic small molecule \\
Structure & Known sequence, variable three-dimensional structure and glycosylation & Well-defined structure \\
Molecular weight & $>1 \mathrm{kDa}$ & $<700 \mathrm{Da}$ \\
Stability & Protease and heat-sensitive & Mostly stable \\
Administration & Parenteral & Oral \\
In vivo half-life & (Usually) Long & (Usually) Short \\
Target & Extracellular & Intracellular \\
Mechanism of action & (Usually) Blocking, depletion & (Usually) Enzyme inhibition \\
Specificity & High & Low/variable \\
Manufacturing cost & High & Low/variable \\
Degradation & Catabolism & Metabolism \\
Generics & Biosimilar & Identical
\end{tabular}

The table highlights selected typical key characterisitics of biologic therapeutics and small-molecule anti-rheumatic agents. Specific drugs may deviate from the typical features. Further differences, such as costs and standards of manufacturing, regulatory requirements, registration path, supply chain logistics, and so on have been omitted.

[6]. Importantly, tyrosine kinases, such as Jak-family kinases [7], the Syk tyrosine kinase [8] or Src-family kinases [9], are also critically involved in immunological processes leading to the development of autoimmune diseases. This has led to the development of small-molecule inhibitors against various protein and lipid kinases for the treatment of autoimmune and inflammatory diseases.

One of the first kinase inhibitors to be developed for rheumatoid arthritis was tofacitinib, a highly selective inhibitor for Jak-family kinases, intracellular molecules involved in signal transduction by various cytokine receptors [7]. Tofacitinib was approved by the Food and Drug Administration (FDA) for the treatment of rheumatoid arthritis in late 2012 (Figure 1), as the first oral anti-rheumatic therapy since the era of biologic agents [10]. Fostamatinib, an inhibitor of the Syk tyrosine kinase which is involved in signaling by various receptors on hematopoietic lineage cells [8], also showed promising therapeutic effects in Phase IIb clinical trials [11] although its further clinical development is in question due to disappointing Phase III trials. Despite initial enthusiasm, targeting the p38 MAP kinase pathway did not prove to be a viable therapeutic option in rheumatoid arthritis [12]. In addition, a number of other small molecule anti-rheumatic agents are in advanced clinical trials for the treatment of rheumatoid arthritis [13]. Although kinase inhibitors are among the most widely explored anti-inflammatory drug candidates, small molecules acting on non-kinase targets such as calcineurin, mTOR, adenosine receptors or ion channels may also provide significant therapeutic benefit.

While a vast knowledge on biologic agents has been obtained during the last 15 years, we are just beginning to collect significant clinical experience with smallmolecule kinase inhibitors. It is already clear that smallmolecule inhibitors have their own unique features related to targets, route of administration, specificity or manufacturing, all of which are quite different from biologic therapeutics (Table 1). Nevertheless, both groups have major benefits and the outcome of their long-term comparison will be one of the most exciting questions of clinical rheumatology in the next several years.

\section{Discussion}

\section{Beneficial aspects of biologic therapies}

Biologic therapies have successfully been introduced into the treatment of several inflammatory rheumatic diseases, including rheumatoid arthritis (RA), ankylosing spondylitis (AS), psoriatic arthritis (PsA), SLE, juvenile idiopathic arthritis (JIA), osteoporosis and ANCA-associated vasculitis. Currently, monoclonal antibodies or fusion proteins targeting TNF- $\alpha$, interleukin- 6 receptor (IL-6R), B-lymphocytes (the CD20 cell surface marker), interleukin-1 (IL-1), Blymphocyte stimulator (BLyS), and CD28-CD80/CD86 co-stimulation have been registered for the treatment of patients with inflammatory rheumatic diseases refractory to conventional immunosuppressive treatment, and the list of promising targets and new indications seems to be growing rapidly $[2,3]$.

\section{Established long-term efficacy}

A key argument in favor of biologics is their outstanding efficacy that has revolutionized the treatment of the abovementioned diseases, leading to novel treatment paradigms. Every novel anti-rheumatic drug is benchmarked against the high standards set by biologics. 
Anti-TNF therapy significantly improves the disease activity of RA, AS or PsA in the majority of the patients compared with conventional DMARDs [14,15], although the benefits become evident only when the biologic is combined with methotrexate, apart from the IL-6R blocker tocilizumab, which appears to be superior to methotrexate even in monotherapy [16]. As many as $40 \%$ of the antiTNF-treated patients can reach a complete remission of the disease, and the benefits of the drugs persist at least as long as five years in the majority of the patients [15]. Long-term remission and even drug-free remission has become a reality in selected patients, especially if the active treatment is started early in the disease course [17]. Consequently, treatment guidelines and diagnostic criteria have recently been updated in order to facilitate early diagnosis and tight control of disease activity [18]. In addition, biologics have been consistently found to reduce, or often halt, the radiographic progression of $\mathrm{RA}$, to preserve the functional status of the involved joints, to reduce fatigue, to control extra-articular manifestations (including accelerated atherosclerosis, the major cause of death in RA patients) and to enable the patients to preserve their ability to work. As all these benefits have been proven to persist during several years of follow-up, the cost-effectiveness of these otherwise expensive drugs has been calculated as acceptable.

\section{Biologics are highly selective for their target molecules}

The therapeutic effect of biologics relies on highly specific protein-protein interactions and, therefore, they are highly selective for their targets. It has been emphasized that specific targeting of a single molecule that has a key pathogenic role in a particular disease increases the efficacy of the drug and reduces the risk of side effects. Oral Jak inhibitors, on the other hand, have multiple targets, as listed in Table 2. Tofacitinib has been shown to block Jak1, Jak3 and, to a lesser extent, Jak2 [19]. While a balanced blockade of several cytokines listed in Table 2 may prove to be beneficial, the activity of a number of other biologically active agents is also modulated by tofacitinib (Table 2). These include haematopoietic factors, growth hormone and growth factors, and substances interfering with lipid metabolism, energy homeostasis, vasoregulation and haemostasis. Some of these interferences (for example, anemia) may have clinical consequences as indicated by data from randomized clinical trials using tofacitinib in which neutropenia and anemia (usually mild) occurred more often in the tofacitinib-treated patients than in the placebo group [20]. These effects, likely related to the broad targeting of Jaks, require clinical monitoring. In addition to adverse effects related to other biological functions of the primary targets (for example, Jak kinases), off-target effects may also represent a major problem for smallmolecule inhibitors. There are, however, strategies that may predict off-target effects in an early phase of drug
Table 2 The use of Jak-family kinases by cytokines and other intercellular mediators

\begin{tabular}{|c|c|}
\hline Ligand & Jak-kinase \\
\hline $\begin{array}{l}\text { IL-6, IL-11, CNTF, CT-1, LIF, OSM, IL-27 } \\
(\text { EBI3 + p28), IL-31, IL-35 (p35 + EBI3) }\end{array}$ & Jak1, Jak2, Tyk2 \\
\hline G-CSF, IL-12 (p40 + p35), angiotensin & Jak2, Tyk2 \\
\hline $\begin{array}{l}\text { Leptin, GM-CSF, IL-5, IL-3, IL-23 (p40 + p19), } \\
\text { serotonin, a-thrombin }\end{array}$ & Jak2 \\
\hline Chemokines & Jak2, Jak3 \\
\hline$\| \mathrm{L}-2$ & Jak1, Jak2, Jak3 \\
\hline IL-4, IL-9, IL-7, IL-15, IL-21 & Jak1, Jak3 \\
\hline IL-13 & Jak1, Jak2, Tyk2 \\
\hline IL-19, IL-20 & Jak1, ? \\
\hline $\begin{array}{l}\text { IL-22, IL-26, IL-28A, IL-28B, IL-29, } \\
\text { interferon (IFNa/ } 3) \text {, IL-10 }\end{array}$ & Jak1, Tyk2 \\
\hline IL-24 & Jak1, ? \\
\hline GH, Epo & Jak2 \\
\hline Thrombopoetin & Jak2, Tyk2 \\
\hline IFN- $\gamma$, PDGF & Jak1, Jak2 \\
\hline TLSP & Jak1, possibly Jak2 \\
\hline EGF & Jak1 \\
\hline
\end{tabular}

Substances that may be involved in off-target effects of Jak-family inhibitors are highlighted in bold. CNTF, ciliary neurotrophic factor; CT-1, cardiotrophin-1; EGF, epidermal growth factor; Epo, erythropoietin; G-CSF, granulocyte colony stimulating factor; $\mathrm{GH}$, growth hormone; GM-CSF, granulocyte-macrophage colony stimulating factor; IL, interleukin; LIF, leukemia inhibitory factor; OSM, oncostatin-M; PDGF, platelet-derived growth factor; TLSP, thymic stromal lymphopoietin.

development, such as genome-wide transcriptome analysis, other in vitro and in vivo assays or new design of future clinical trials [21-23].

Of note, anemia was also more common in the adalimumab-treated subjects [24]. Clinically significant neutropenia and associated infection are rare with antiTNF therapies and also with rituximab, but the regular control of blood count is advisable. In contrast, neutropenia occurs relatively frequently during the IL-6R blocker therapy (with a frequency of $29 \%$ and $33 \%$ in two randomized controlled trials (RCTs) $[16,25]$, but high-grade neutropenia or significant infectious events are rare. Hemoglobin levels typically normalize quickly after the initiation of anti-TNF therapy, and even faster during tocilizumab treatment.

\section{Beneficial cardiovascular effects of biologics}

Anti-TNF agents have proven to reduce all-cause cardiovascular morbidity and mortality [26]. This effect is likely linked to changes in lipid metabolism; however, its mechanism is currently not fully understood. Total cholesterol, as well as both low-density lipoprotein (LDL) and highdensity lipoprotein (HDL) levels typically decrease during an active inflammatory process in rheumatoid arthritis, but rise again once the acute phase response is suppressed by an effective therapy [27]. In this context, lipid levels 
display an inverse correlation with C-reactive protein (CRP) levels, a widely used marker of acute phase response. In fact, a persistently elevated CRP level has been found to closely correlate with cardiovascular risk, and the normalization of CRP in response to therapy is an indicator of lower atherogenic risk. Some investigators have found levels of LDL to rise and HDL to decrease during infliximab therapy [28], a phenomenon called the 'lipid paradox' (indicating reduced cardiovascular risk despite an increased LDL to HDL ratio) [27], whereas others have demonstrated that HDL levels and the atherogenic index are unchanged. More detailed analyses have revealed that the Apo B/A-I ratio improves and macrophage inhibitory factor levels decrease during adalimumab treatment, and that the capacity of HDL to block the oxidation of LDL and paraoxonase-1 activity increases during anti-TNF therapy [29]. All of these findings support the beneficial effects of anti-TNF treatment on lipid metabolism and atherosclerotic risk.

TNF- $\alpha$ is also known to cause endothelial activation, including the up-regulation of adhesion molecules, and it increases the production of coagulation factors and enhances platelet activation [30]. Endothelial dysfunction is seen as a major pathogenic factor in RA-associated cardiovascular morbidity with an important role for TNF- $\alpha$ $[31,32]$. TNF- $\alpha$ also contributes to insulin resistance, a phenomenon that occurs more often in RA patients than in matched healthy subjects or osteoarthritis patients [33].

Both tocilizumab, a biologic agent targeting IL-6, and the small molecule tofacitinib have been shown to significantly affect total cholesterol, LDL and HDL levels, although the overall effect on cardiovascular risk has yet to be determined $[24,34,35]$. Findings suggest that tocilizumab could improve the arterial stiffness with comparable efficacy to anti-TNF agents; however, the net effect of tocilizumab and tofacitinib on long-term cardiovascular morbidity has yet to be elucidated.

\section{Suitability for renal or hepatic impaired patients}

Biologic therapies can be administered in patients with seriously impaired renal function. No differences have been found in the pharmacokinetics of etanercept between patients on hemodialysis and those with normal renal function. Obviously, these agents are not removed by hemodialysis or peritoneal dialysis, and, consequently, they have been found to be safe and effective in these patients [36]. In contrast, there are no such data available for novel small molecules, such as tofacitinib, in patients with severe renal failure. The dose of tofacitinib must be reduced in patients with moderate renal insufficiency according to the current FDA label. A proportion of patients with impaired renal function may not be appropriate candidates for Jak-inhibitor therapy.

Liver enzyme elevations ( $>3 \times$ upper limit of normal) were more common with tofacitinib than with adalimumab therapy [20]. Abnormal liver function is not a contraindication to anti-TNF or rituximab treatment, and hepatic enzyme elevation is rare, usually mild and transient during these therapies. In contrast, the dose of tocilizumab should be reduced in patients with moderate liver enzyme elevation, and the drug is not recommended for patients with severe hepatic impairment according to the FDA label. It should be noted that similar restrictions apply to the use of the IL-6R blocker tocilizumab, as this agent also causes liver enzyme elevations in a significant proportion of patients.

\section{Intravenous infusion ensures maximum compliance}

It has been stressed that one potential advantage of small molecules is their oral administration route; however, arguments may also favor parenteral administration. Studies comparing intravenous and oral bisphosphonate anti-osteoporotic regimens have suggested a poorer compliance with once weekly oral administration as compared with a tri-monthly or yearly intravenous route, which may partly be responsible for the lower efficacy of the oral agents [37]. A better compliance may be expected with injected therapies than with twice daily tablets in a certain proportion of patients. Apart from compliance, parenteral administration of methotrexate has been shown not only to cause fewer gastrointestinal adverse effects but also to have higher efficacy and a faster onset of action [38].

\section{Biologics may be well suited for individualized therapy}

As RA and most of the autoimmune rheumatic diseases are also of a heterogeneous nature in terms of therapeutic response, the identification of biomarkers with a potential to predict the response to the particular agent is of high priority. Based on theoretical considerations, differential responses to drugs targeting one single molecule may better identify disease subsets than a molecule with pleiotropic activity. Several parameters have been identified with a potential to predict response to anti-TNF or B-cell-depleting therapies [39], and some of the identified biomarkers (such as genetic polymorphisms or levels of autoantibodies) may prove to be useful in the identification of patient populations with optimal response to biological therapies. However, it should be noted that truly predictive biomarkers have so far mainly been identified for small-molecule inhibitors in oncology; therefore, small molecule inhibitors may be equally well suited for the individualized therapy of inflammatory diseases.

\section{Beneficial aspects of small-molecule therapeutics}

The introduction of biologics and substantial additional efforts aimed at understanding autoimmunity and inflammation at the molecular level has not only revolutionized the therapy of rheumatic diseases but facilitated the identification of key pathogenic molecules in autoimmune diseases. A better understanding of the molecular nature 
of immune cell receptors as well as the intracellular signaling pathways downstream of these receptors has led to novel approaches to target various players in disease pathogenesis. Until recently, however, the success of small molecule targeted therapeutic approaches in rheumatology has not achieved the level of biologics, mainly due to off-target toxicity (non-selectivity), inefficacy, or unfavorable risk/benefit ratios. This is in contrast to the experience in cancer therapy where small molecule-based targeted therapies (such as BCR-Abl inhibitors) have been used successfully for the treatment of chronic myeloid leukemia and other hematological malignancies.

How can small molecules contribute to the therapeutic armamentarium of rheumatic diseases that is currently dominated by biologics? For this, one should take into account the key differences between small molecules and biologics (Table 1). In particular, small molecules: 1) are typically administered orally; 2) have a higher likelihood for off-target effects (based on non-selectivity); and 3) have the ability to directly target intracellular signaling pathways. This section reviews these three key points to underscore why novel small molecules may also have a promising future in rheumatology.

Oral administration is a major advantage for small molecules Despite the outstanding efficacy and good safety/tolerability profile of the currently used biologics in rheumatology, the requirement for long-term injections or infusions can impose a burden on patients leading to reduced adherence. Needle phobia is not uncommon: twenty-two percent of participants in the general population reported a fear of needles in a recent survey [40]. In multiple sclerosis, for which no effective oral treatment was available until recently, injection anxiety or needle phobia could prevent patients from self-injecting their biologics [41]. Misunderstanding about the risks of self-injection or a lack of knowledge about how best to manage injection pain and side effects may also result in an inability to self-inject [41].

Advances in injection technology, such as the introduction of auto-injectors have improved patient satisfaction and reduced the incidence of injection-site reactions; however, patients' resistance to self-injection still exists [41]. According to the RAISE (Rheumatoid Arthritis: Insights, Strategies and Expectations, 2009) survey, about 25\% of survey participants with RA currently treated with a subcutaneous injection rely on caregivers or healthcare providers to administer the medication, and $24 \%$ of patients who self-inject experience pain upon injection and $20 \%$ experience irritation at the injection site [42]. Injection-site reactions or patients' negative feelings towards any therapy administered via needles may negatively affect patient acceptance of treatment and often result in non-compliance.

Non-adherence to a treatment regimen is a prevalent and major problem of patients with chronic disorders.
Approximately half of the patients with a chronic disease have problems following their prescribed regimen to the extent that they are unable to obtain the desired clinical benefit, and poor adherence attenuates optimal clinical benefit [43]. The World Health Organization has recognized the lack of adherence as a major problem in the management of chronic diseases and concluded that improving adherence would have an even more beneficial effect on health outcome than improving the efficacy of specific treatments [44]. It is often assumed that the population is generally adherent but in many chronic conditions, on average, only about half of the patients comply with care recommendations over the long term [45].

Overall, oral therapies with new modes of action have the potential to expand the current treatment repertoire, increase patient satisfaction and adherence, and thereby improve efficacy. Patients express greater satisfaction with the convenience of oral therapies, and oral medications are known to be preferred to injected therapies that have similar efficacy. Thus, treatment efficacy can be improved simply through its effect on adherence.

\section{Broader target selectivity can be of therapeutic benefit for small molecules}

The experience in cancer therapy established the principle that kinase inhibitors do not need to be absolutely specific to be clinically useful; off-target effects may even be therapeutically beneficial. The competitive inhibition of the BCR-Abl tyrosine kinase leads to inhibition of proliferation, restoration of cell cycle control, induction of apoptosis and reversal of genetic instability in BCR-Abl dependent cells in vitro [46]. Based on this mode of action, BCR-Abl inhibitors such as imatinib or next generation sunitinib, dasatinib and nilotinib can induce remission in more than $90 \%$ of patients in the early stages of chronic myelogenous leukemia (CML). Imatinib, however, is not selective for BCR-Abl and also inhibits the PDGFR kinase and KIT receptor tyrosine kinase, thereby expanding its therapeutic utility to other diseases, for example, gastrointestinal stromal tumors [47] or idiopathic hypereosinophilic syndrome [48]. In addition, the effect of dasatinib is likely, at least in part, mediated by its inhibitory effect on Src-family kinases. Further potential benefit of broad(er) targeting may be the simultaneous modulation of several cytokine pathways in the same disease (for example, by Jak inhibition in RA) exerting a stronger therapeutic effect (Table 2).

Small molecules are often associated with adverse reactions due to off-target effects. However, biologics with very high specificity can also cause severe adverse events. For example, an increased risk of serious tuberculosis and other opportunistic infections has been reported with TNF-blocking agents across various studies, and that effect is likely directly related to the target molecule [49]. In addition, the effect of certain biologics 
(such as the B-cell-depleting rituximab) is mediated by depleting the cellular lineage expressing the target molecule; in that case, a wider functional consequence of biological therapy is expected. Small-molecule Jak inhibitors have been shown to adversely impact serum lipid profiles; however, pro-atherogenic lipid changes and decreased hepatic LDL receptor expression were also demonstrated with tocilizumab in RA [34]. The net effect of these drugs on morbidity and mortality has to be confirmed in future long term clinical trials regardless of whether it is related to on- or off-target effects.

Overall, for the patient and treating physician, the risk/ benefit will be determined by both off-target and on-target effects and is based on safety and efficacy - regardless of target selectivity.

\section{Inhibiting intracellular signaling may be as effective as targeting specific cell surface receptors}

Inhibition of intracellular signaling molecules operating at critical nodes of pathways has always been an attractive approach to achieve a high magnitude and/or robust duration of therapeutic response in inflammatory immune mediated diseases [50]. Small molecules can directly target such intracellular pathways for which a large variety of candidate molecules exists; of those, kinases still remain the most attractive targets in rheumatology. Just as the biologic era came about as a consequence of major advances in protein engineering to allow the production of antibodies or engineered derivatives, advances in medicinal chemistry in the past two decades have also enabled the otherwise challenging development of very specific kinase inhibitors as therapeutic agents.

Kinases catalyze reversible phosphorylation, a fundamental mechanism for the regulation of protein function in various receptor-mediated processes, such as cell growth and differentiation in eukaryotic cells [51]. Many key immune receptors, including those responsible for driving inflammation, exert their effects through kinases and phosphorylation events. The human kinome comprises a total of 518 kinases and a large fraction of those functions downstream of cytokine, antigen and Fc-receptors, all representing potential targets for the treatment of rheumatic diseases.

More than 60 cytokines signal via the Janus kinase (Jak) and signal transducer and activator of transcription (STAT) pathways. Jak inhibitors have now been approved for the treatment of immune-mediated diseases, such as RA and myelofibrosis, and various Jak inhibitors are being developed and tested in a wide range of autoimmune diseases [52]. Although long term safety and efficacy data are not yet available for these inhibitors, the short term efficacy of tofacitinib, a Jak inhibitor in RA seems to be comparable to that of anti-TNF agents while side effects, such as infection, anemia and neutropenia, appear to be directly related to its mode of action [53].
Beyond Jaks, spleen tyrosine kinase (Syk) and Bruton tyrosine kinase (Btk) are also critical enzymes in the signaling pathways activated by immunological receptors involved in $\mathrm{B}$ and $\mathrm{T}$ cell function [50]. Kinases downstream of antigen and Fc-receptors also represent novel targets including members of the protein kinase $\mathrm{C}$ (PKC) family, mitogen-activated protein kinase (MAPK) family, the lipid kinase phosphoinositide 3-kinase (PI3K), protein kinase B ( $\mathrm{PKB}$, also known as Akt) and mammalian target of rapamycin (mTOR). Inhibitors of these proteins are currently being tested in preclinical models or clinical trials of various immune-mediated diseases [50]. Development of oral inhibitors of Jak and Syk are the most advanced and have already demonstrated clinical efficacy in RA.

Furthermore, not only kinases can be targeted by oral immune modulators. Examples include early studies in RA with a sphingosine-1-phosphate lyase inhibitor to modulate lymphocyte redistribution, or with the chemokine receptor-1 antagonist involved in osteoclast maturation, mobility and activation [54].

Given the large number of key effector proteins discovered in a wide variety of cell functions, together with the growing scientific understanding of the pathogenesis of immune mediated diseases, further small molecule based targeted immunomodulators will likely emerge in the near future.

\section{Additional potential benefits of small molecules}

Biologic agents and small molecules usually differ in their half-life, as well. Protein-like biologics currently used or tested in rheumatology usually have a longer half-life than small molecules. While such biologics require less frequent administration, they also need longer elimination; in cases where fast elimination is desirable, for example, during infections or before surgical interventions, the short half-life of small molecules may be of considerable clinical benefit.

Beyond the medical aspects, there are other factors that may have a positive effect on the overall cost-effectiveness of small molecules. Although it is too early to speculate about the long-term costs of small molecules, should these agents (requiring less complex and less expensive manufacturing) be significantly less expensive than biologics, this would certainly add significant value to the use of small molecules. Some small molecule drugs are also advantageous in terms of several logistical aspects, such as longer shelf life, no need for refrigeration and easier distribution in rural areas where infrastructure/experience with parenteral administration is limited.

\section{Summary}

After a revolution in rheumatology by biologic therapy, novel intracellular targets have also emerged from an improved understanding of the immunopathogenesis of inflammatory rheumatic diseases. While biologics can 
modulate extracellular protein-protein interactions, intracellular processes can be more effectively targeted by small molecules. As a result, a number of small-molecule anti-rheumatic agents have been developed and many of those have progressed to preclinical stages or human clinical studies. As compared to biologics, small molecules also differ in the route of administration, target selectivity and specificity in terms of safety and efficacy as well as development path and overall cost. Despite such major advances in rheumatology, still no ideal treatment exists as current therapies are not causal and no single monotherapy, either small molecule or biologic, is capable of inducing full remission in the majority of patients in most rheumatic diseases. True synergies exist when both classic small molecule approaches and protein therapeutic strategies are simultaneously applied. Combination therapy likely continues to be a key therapeutic approach for most rheumatic diseases.

Currently, several novel approaches with biologics as well as small molecules are being tested to target various immunopathogenic players in immune-mediated rheumatic diseases. In addition to the existing effective biologic agents, highly effective oral drugs also seem to emerge. The next several years will witness a real-life comparison of the clinical efficacy and safety of biologics and oral therapies in rheumatology and provide the answers eagerly awaited by the rheumatology community.

\section{Competing interests}

Peter Gergely is an employee of Novartis. The other two authors declare that they have no competing interests.

\section{Authors' contributions}

All three authors contributed to the conception and design of the study, helped to draft the manuscript, and read and approved the final manuscript.

\section{Acknowledgements}

Work in the authors' groups was supported by the European Research Council (Starting Independent Investigator Award No. 206283 to A. M.), the Wellcome Trust (International Senior Research Fellowship No. 087782 to A. M.), the European Union's 7th Framework Programme (TARKINAID project to A. M.), the 'Lendület' program of the Hungarian Academy of Sciences (LP2013-66/2013 to A. M.), the Hungarian National Development Agency (TÁMOP 4.2.2.A-II/I/KONV-2012-0035 and TÁMOP 4.2.2.B to L. K.) and Novartis (P. G.). The authors would like to thank David Lee (Novartis) and Tamás Németh (Semmelweis University) for their critical review of the manuscript. This work was presented at the International Congress on Controversies in Rheumatology and Autoimmunity (CORA) Meeting, Budapest, Hungary, April 4th, 2013.

\footnotetext{
Author details

'Department of Physiology, Semmelweis University School of Medicine, 1094 Budapest, Hungary. "MTA-SE "Lendület" Inflammation Physiology Research Group of the Hungarian Academy of Sciences and the Semmelweis University, 1094 Budapest, Hungary. ${ }^{3}$ Department of Rheumatology, University of Szeged Faculty of Medicine, 6725 Szeged, Hungary. ${ }^{4}$ Translational Medicine Autoimmunity, Novartis Institutes for Biomedical Research, $\mathrm{CH}-4002$ Basel, Switzerland.
}

Received: 14 November 2013 Accepted: 19 February 2014 Published: 13 Mar 2014

\section{References}

1. Feldmann M: Development of anti-TNF therapy for rheumatoid arthritis. Nat Rev Immunol 2002, 2:364-371

2. Choy EH, Kavanaugh AF, Jones SA: The problem of choice: current biologic agents and future prospects in RA. Nat Rev Rheumatol 2013, 9:154-163.

3. Navarro-Millan I, Curtis JR: Newest clinical trial results with antitumor necrosis factor and nonantitumor necrosis factor biologics for rheumatoid arthritis. Curr Opin Rheumatol 2013, 25:384-390.

4. Druker BJ: Translation of the Philadelphia chromosome into therapy for CML. Blood 2008, 112:4808-4817.

5. Arora A, Scholar EM: Role of tyrosine kinase inhibitors in cancer therapy. J Pharmacol Exp Ther 2005, 315:971-979.

6. Grimminger F, Schermuly RT, Ghofrani HA: Targeting non-malignant disorders with tyrosine kinase inhibitors. Nat Rev Drug Discov 2010, 9:956-970.

7. Ghoreschi K, Laurence A, O'Shea JJ: Janus kinases in immune cell signaling. Immunol Rev 2009, 228:273-287.

8. Mócsai A, Ruland J, Tybulewicz VL: The SYK tyrosine kinase: a crucial player in diverse biological functions. Nat Rev Immunol 2010, 10:387-402

9. Lowell CA: Src-family kinases: rheostats of immune cell signaling. $\mathrm{Mol}$ Immunol 2004, 41:631-643.

10. van Vollenhoven RF: Rheumatoid arthritis in 2012: progress in RA genetics, pathology and therapy. Nat Rev Rheumatol 2012, 2013:70-72.

11. Weinblatt ME, Kavanaugh A, Genovese MC, Musser TK, Grossbard EB, Magilavy DB: An oral spleen tyrosine kinase (Syk) inhibitor for rheumatoid arthritis. N Engl J Med 2010, 363:1303-1312.

12. Hammaker D, Firestein GS: "Go upstream, young man": lessons learned from the p38 saga. Ann Rheum Dis 2010, 69:i77-i82.

13. van Vollenhoven RF: Small molecular compounds in development for rheumatoid arthritis. Curr Opin Rheumatol 2013, 25:391-397.

14. van der Heijde D, Klareskog L, Rodriguez-Valverde V, Codreanu C, Bolosiu H, Melo-Gomes J, Tornero-Molina J, Wajdula J, Pedersen R, Fatenejad S, Investigators TS: Comparison of etanercept and methotrexate, alone and combined, in the treatment of rheumatoid arthritis: two-year clinical and radiographic results from the TEMPO study, a double-blind, randomized trial. Arthritis Rheum 2006, 54:1063-1074.

15. Braun J, Baraliakos X, Listing J, Fritz C, Alten R, Burmester G, Krause A, Schewe S, Schneider M, Sorensen H, Zeidler H, Sieper J: Persistent clinical efficacy and safety of anti-tumour necrosis factor alpha therapy with infliximab in patients with ankylosing spondylitis over 5 years: evidence for different types of response. Ann Rheum Dis 2008, 67:340-345.

16. Jones G, Sebba A, Gu J, Lowenstein MB, Calvo A, Gomez-Reino JJ, Siri DA, Tomsic M, Alecock E, Woodworth T, Genovese MC: Comparison of tocilizumab monotherapy versus methotrexate monotherapy in patients with moderate to severe rheumatoid arthritis: the AMBITION study. Ann Rheum Dis 2010, 69:88-96.

17. Saleem B, Keen H, Goeb V, Parmar R, Nizam S, Hensor EM, Churchman SM, Quinn M, Wakefield R, Conaghan PG, Ponchel F, Emery P: Patients with RA in remission on TNF blockers: when and in whom can TNF blocker therapy be stopped? Ann Rheum Dis 2010, 69:1636-1642.

18. Smolen JS, Aletaha D, Bijlsma JW, Breedveld FC, Boumpas D, Burmester G, Combe B, Cutolo M, de Wit M, Dougados M, Emery P, Gibofsky A, Gomez-Reino JJ, Haraoui B, Kalden J, Keystone EC, Kvien TK, McInnes I, Martin-Mola E, Montecucco C, Schoels M, van der Heijde D, T2T Expert Committee: Treating rheumatoid arthritis to target: recommendations of an international task force. Ann Rheum Dis 2010, 69:631-637.

19. Ghoreschi K, Jesson MI, Li X, Lee JL, Ghosh S, Alsup JW, Warner JD, Tanaka M, Steward-Tharp SM, Gadina M, Thomas CJ, Minnerly JC, Storer CE, LaBranche TP, Radi ZA, Dowty ME, Head RD, Meyer DM, Kishore N, O'Shea JJ: Modulation of innate and adaptive immune responses by tofacitinib (CP-690,550). J Immunol 2011, 186:4234-4243.

20. Fleischmann R, Kremer J, Cush J, Schulze-Koops H, Connell CA, Bradley JD, Gruben D, Wallenstein GV, Zwillich SH, Kanik KS, ORAL Solo Investigators: Placebo-controlled trial of tofacitinib monotherapy in rheumatoid arthritis. N Engl J Med 2012, 367:495-507.

21. Hidalgo M, Eckhardt SG: Development of matrix metalloproteinase inhibitors in cancer therapy. J Natl Cancer Inst 2001, 93:178-193.

22. Ospelt C, Gay S: Safety concerns on the development of novel therapeutic drugs. Arthritis Res Ther 2006, 8:112.

23. Flammer AJ, Sudano I, Hermann F, Gay S, Forster A, Neidhart M, Kunzler P, Enseleit F, Periat D, Hermann M, Nussberger J, Luscher TF, Corti R, Noll G, 
Ruschitzka F: Angiotensin-converting enzyme inhibition improves vascular function in rheumatoid arthritis. Circulation 2008, 117:2262-2269.

24. van Vollenhoven RF, Fleischmann R, Cohen S, Lee EB, Garcia Meijide JA, Wagner S, Forejtova S, Zwillich SH, Gruben D, Koncz T, Wallenstein GV, Krishnaswami S, Bradley JD, Wilkinson B, ORAL Standard Investigators: Tofacitinib or adalimumab versus placebo in rheumatoid arthritis. N Engl J Med 2012, 367:508-519.

25. Genovese MC, McKay JD, Nasonov EL, Mysler EF, da Silva NA, Alecock E, Woodworth T, Gomez-Reino JJ: Interleukin-6 receptor inhibition with tocilizumab reduces disease activity in rheumatoid arthritis with inadequate response to disease-modifying antirheumatic drugs: the tocilizumab in combination with traditional disease-modifying antirheumatic drug therapy study. Arthritis Rheum 2008, 58:2968-2980.

26. Dixon WG, Watson KD, Lunt M, Hyrich KL, British Society for Rheumatology Biologics Register Control Centre Consortium, Silman AJ, Symmons DP, British Society for Rheumatology Biologics Register: Reduction in the incidence of myocardial infarction in patients with rheumatoid arthritis who respond to anti-tumor necrosis factor alpha therapy: results from the British Society for Rheumatology Biologics Register. Arthritis Rheum 2007, 56:2905-2912

27. Myasoedova E, Crowson CS, Kremers HM, Roger VL, Fitz-Gibbon PD, Therneau TM, Gabriel SE: Lipid paradox in rheumatoid arthritis: the impact of serum lipid measures and systemic inflammation on the risk of cardiovascular disease. Ann Rheum Dis 2011, 70:482-487.

28. Popa C, van den Hoogen FH, Radstake TR, Netea MG, Eijsbouts AE, den Heijer M, van der Meer JW, van Riel PL, Stalenhoef AF, Barrera P: Modulation of lipoprotein plasma concentrations during long-term anti-TNF therapy in patients with active rheumatoid arthritis. Ann Rheum Dis 2007, 66:1503-1507.

29. Popa C, van Tits LJ, Barrera P, Lemmers HL, van den Hoogen FH, van Riel PL, Radstake TR, Netea MG, Roest M, Stalenhoef AF: Anti-inflammatory therapy with tumour necrosis factor alpha inhibitors improves high-density lipoprotein cholesterol antioxidative capacity in rheumatoid arthritis patients. Ann Rheum Dis 2009, 68:868-872.

30. Heathfield SK, Parker B, Zeef LA, Bruce IN, Alexander MY: Certolizumab pegol attenuates the pro-inflammatory state in endothelial cells in a manner that is atheroprotective. Clin Exp Rheumatol 2013, 31:225-233.

31. McKellar GE, McCarey DW, Sattar N, McInnes IB: Role for TNF in atherosclerosis? Lessons from autoimmune disease. Nat Rev Cardiol 2009, 6:410-417.

32. Khan F, Galarraga B, Belch JJ: The role of endothelial function and its assessment in rheumatoid arthritis. Nat Rev Rheumatol 2010, 6:253-261.

33. Stagakis I, Bertsias G, Karvounaris S, Kavousanaki M, Virla D, Raptopoulou A, Kardassis D, Boumpas DT, Sidiropoulos PI: Anti-tumor necrosis factor therapy improves insulin resistance, beta cell function and insulin signaling in active rheumatoid arthritis patients with high insulin resistance. Arthritis Res Ther 2012, 14:R141.

34. Strang AC, Bisoendial RJ, Kootte RS, Schulte DM, Dallinga-Thie GM, Levels JH, Kok M, Vos K, Tas SW, Tietge UJ, Müller N, Laudes M, Gerlag DM, Stroes ES, Tak PP: Pro-atherogenic lipid changes and decreased hepatic LDL receptor expression by tocilizumab in rheumatoid arthritis. Atherosclerosis 2013, 229:174-181

35. McInnes IB, Thompson L, Giles JT, Bathon JM, Salmon JE, Beaulieu AD, Codding CE, Carlson TH, Delles C, Lee JS, Sattar N: Effect of interleukin- 6 receptor blockade on surrogates of vascular risk in rheumatoid arthritis: MEASURE, a randomised, placebo-controlled study. Ann Rheum Dis 2013. doi:10.1136/annrheumdis-2013-204345.

36. Senel S, Kisacik B, Ugan Y, Kasifoglu T, Tunc E, Cobankara V: The efficacy and safety of etanercept in patients with rheumatoid arthritis and spondyloarthropathy on hemodialysis. Clin Rheumatol 2011, 30:1369-1372.

37. Ziller V, Kostev K, Kyvernitakis I, Boeckhoff J, Hadji P: Persistence and compliance of medications used in the treatment of osteoporosis-analysis using a large scale, representative, longitudinal German database. Int J Clin Pharmacol Ther 2012, 50:315-322.

38. Braun J, Kastner P, Flaxenberg P, Wahrisch J, Hanke P, Demary W, von Hinuber U, Rockwitz K, Heitz W, Pichlmeier U, Guimbal-Schmolck C, Brandt A, MC-MTX.6/RH Study Group: Comparison of the clinical efficacy and safety of subcutaneous versus oral administration of methotrexate in patients with active rheumatoid arthritis: results of a six-month, multicenter, randomized, double-blind, controlled, phase IV trial. Arthritis Rheum 2008, 58:73-81.

39. Emery $\mathrm{P}$, Dorner T: Optimising treatment in rheumatoid arthritis: a review of potential biological markers of response. Ann Rheum Dis 2011, 70:2063-2070
40. Wright $\mathrm{S}$, Yelland $\mathrm{M}$, Heathcote $\mathrm{K}, \mathrm{Ng} \mathrm{SK}$, Wright $\mathrm{G}$ : Fear of needles-nature and prevalence in general practice. Aust Fam Physician 2009, 38:172-176.

41. Cohen BA, Rieckmann P: Emerging oral therapies for multiple sclerosis. Int J Clin Pract 2007, 61:1922-1930.

42. McInnes IB, Combe B, Burmester G: Understanding the patient perspective - results of the Rheumatoid Arthritis: Insights, Strategies \& Expectations (RAISE) patient needs survey. Clin Exp Rheumatol 2013, 31:350-357.

43. Dunbar-Jacob J, Erlen JA, Schlenk EA, Ryan CM, Sereika SM, Doswell WM: Adherence in chronic disease. Annu Rev Nurs Res 2000, 18:48-90.

44. World Health Organization: Adherence to Long-term Therapies: Evidence for Action. 2003. Available at: http:/www.who.int/chp/knowledge/publications/ adherence_introduction.pdf.

45. Sawyer SM, Aroni RA: Sticky issue of adherence. J Paediatr Child Health 2003, 39:2-5.

46. An X, Tiwari AK, Sun Y, Ding PR, Ashby CR Jr, Chen ZS: BCR-ABL tyrosine kinase inhibitors in the treatment of Philadelphia chromosome positive chronic myeloid leukemia: a review. Leuk Res 2010, 34:1255-1268.

47. Demetri $G D$, von Mehren $M$, Blanke $C D$, Van den Abbeele $A D$, Eisenberg $B$, Roberts PJ, Heinrich MC, Tuveson DA, Singer S, Janicek M, Fletcher JA, Silverman SG, Silberman SL, Capdeville R, Kiese B, Peng B, Dimitrijevic S, Druker BJ, Corless C, Fletcher CD, Joensuu H: Efficacy and safety of imatinib mesylate in advanced gastrointestinal stromal tumors. N Engl J Med 2002, 347:472-480.

48. Cools J, DeAngelo DJ, Gotlib J, Stover EH, Legare RD, Cortes J, Kutok J, Clark J, Galinsky I, Griffin JD, Cross NC, Tefferi A, Malone J, Alam R, Schrier SL, Schmid J, Rose M, Vandenberghe P, Verhoef G, Boogaerts M, Wlodarska I, Kantarjian H, Marynen P, Coutre SE, Stone R, Gilliland DG: A tyrosine kinase created by fusion of the PDGFRA and FIP1L1 genes as a therapeutic target of imatinib in idiopathic hypereosinophilic syndrome. $N$ Engl J Med 2003, 348:1201-1214.

49. Jain A, Singh JA: Harms of TNF inhibitors in rheumatic diseases: a focused review of the literature. Immunotherapy 2013, 5:265-299.

50. O'Shea JJ, Laurence A, McInnes IB: Back to the future: oral targeted therapy for RA and other autoimmune diseases. Nat Rev Rheumatol 2013, 9:173-182.

51. Kontzias A, Kotlyar A, Laurence A, Changelian P, O'Shea JJ: Jakinibs: a new class of kinase inhibitors in cancer and autoimmune disease. Curr Opin Pharmacol 2012, 12:464-470.

52. Seavey MM, Dobrzanski P: The many faces of Janus kinase. Biochem Pharmacol 2012, 83:1136-1145.

53. Burmester GR, Blanco R, Charles-Schoeman C, Wollenhaupt J, Zerbini C, Benda B, Gruben D, Wallenstein G, Krishnaswami S, Zwillich SH, Koncz T, Soma K, Bradley J, Mebus C, ORAL Step investigators: Tofacitinib (CP-690,550) in combination with methotrexate in patients with active rheumatoid arthritis with an inadequate response to tumour necrosis factor inhibitors: a randomised phase 3 trial. Lancet 2013, 381:451-460.

54. Fleischmann R: Novel small-molecular therapeutics for rheumatoid arthritis. Curr Opin Rheumatol 2012, 24:335-341.

\subsection{6/1741-7015-12-43}

Cite this article as: Mócsai et al.: What is the future of targeted therapy in rheumatology: biologics or small molecules?. BMC Medicine 2014, 12:43

\section{Submit your next manuscript to BioMed Central and take full advantage of:}

- Convenient online submission

- Thorough peer review

- No space constraints or color figure charges

- Immediate publication on acceptance

- Inclusion in PubMed, CAS, Scopus and Google Scholar

- Research which is freely available for redistribution 